\title{
O “QUADRO GEOGNÓSTICO DO BRASIL" DE WILHELM LUDWIG VON ESCHWEGE: BREVES COMENTARIOS A SUA VISÃO DA GEOLOGIA NO BRASIL
}

\author{
Friedrich E. Renger*
}

\begin{abstract}
Resumo
O trabalho apresenta uma resenha da obra "Geognostisches Gemälde von Brasilien und wahrscheinliches Muttergestein der Diamanten " de Wilhelm Ludwig von Eschwege por ocasião do sesquicentenário da sua morte em $1^{\circ}$ de fevereiro de 2005. A obra foi publicada, em pequena tiragem, em 1822. Trata inicialmente dos grandes divisores de água: um de direção aproximada leste - oeste, separando a bacia do rio Amazonas das dos rios Paraná e Paraguai, que Eschwege batiza de "Serra das Vertentes", o outro divisor separa a bacia do Rio São Francisco dos rios que correm diretamente ao Oceano Atlântico o qual chama de "Serra do Espinhaço", incluindo nela a Serra da Mantiqueira. Em segundo lugar apresenta um esquema estratigráfico baseado nos modelos usados na Europa, como, por exemplo, aquele proposto em 1787 por Abraham Gottlieb Werner, professor da Academia de Minas de Freiberg na Alemanha. A Primeira Formação Primitiva é formada pelo embasamento cristalino, a Segunda Formação Primitiva corresponde às seqüências supracrustais dobradas (representadas pelos Supergrupos Rio das Velhas, Minas e Espinhaço), a Terceira ou de Transição abrange essencialmente o atual Grupo Bambui e uma quarta subdivisão reúne depósitos superficiais como aluviões e coberturas terciárias e quarternárias. Percebe-se que suas idéias a respeito da geologia do Brasil são fortemente influenciadas pela escola netunista de Werner. Descreve ainda macroscopicamente os principais tipos de rocha encontrados no Brasil, define os novos termos "itacolumito" e "itabirito" e introduz o termo "tapanhoacanga" na nomenclatura geológica, todos com suas localidadestipo. Tapanhoacanga, hoje reduzida para canga, é de origem indígena de tapanhu = escravo negro e acanga = cabeça (ou a $=$ cabeça e canga $=$ osso). A última parte do "Quadro geognóstico... " trata da ocorrência dos diamantes no Brasil e de sua possível rocha matriz, na sua opinião formados em concreções limoníticas originadas das rochas ferruginosas da Segunda Formação Primitiva.
\end{abstract}

Palavras-chave: História da Geologia, Quadrilátero Ferrífero, Serra do Espinhaço, estratigrafia precambriana, itabirito, itacolumito, canga, diamantes

\begin{abstract}
ESCHWEGE'S “GEOGNOSTICAL SKETCH OF BRAZIL AND THE PROBABLE SOURCE ROCK OF DIAMONDS": BRIEF COMMENTS ON HIS VISION OF BRAZILIAN GEOLOGY. This small brochure was published in 1822 by the German geologist Wilhem Ludwig von Eschwege (1777 - 1855) and is now translated to Portuguese for the first time completely as a memorial of his passing away 150 years ago. Initially, Eschwege reports on the physical geography of Brazil and suggests the names "Serra das Vertentes" (Watershed Mountains) and "Serra do Espinhaço" (Backbone Ridge), running East - West the first and North - South the second, separating the great hydrographic basins in Brazil. A main chapter is dedicated to a very first proposal of a stratigraphic scheme based on European models of the time and heavily influenced by A. G. Werner, the principal protagonist of the neptunism, which interpreted all rocks as being precipitated from aqueous solutions. He distinguishes four stratigraphic divisions: the First Primitive Formation containing granite, gneiss, and mica schist, corresponding in more modern terms to the crystalline basement; the Second Primitive Formation is formed by itacolumite (quartzite), itabirite (iron formation) and schist, represented by the Rio das Velhas, Minas, and Espinhaço supergroups. The third or Transitional Formation composed by slates, quartz schist, greywacke, and massive limestone corresponds to the Macaúbas and Bambui groups. The fourth and uppermost formation encloses all superficial deposits, such as alluvial, river gravels and a peculiar ferruginous conglomerate called by the native tapanhoacanga, which means Negro head. His argumentation is heavily influenced by neptunistic thinking. Eschwege still describes in great detail the principal rock types, as known at this time in Brazil and introduces the terms itacolumite, itabirite and (tapanhoa)canga into the geological nomenclature. The second part is dedicated to the occurrence, distribution and origin of Brazilian diamonds. He considers that they are formed within any rock of his Second Primitive Formation, due to the occasional founding of limonitic concretions with inclusions of diamonds.
\end{abstract}

Keywords: History of geology, Quadrilátero Ferrífero, Serra do Espinhaço, Precambrian stratigraphy, itabirite, itacolumite, canga, diamonds 


\section{INTRODUÇ̃̃o}

O "Quadro Geognóstico do Brasil" foi escrito em 1822 pelo Barão de Eschwege (1777 - 1855) e impresso em pequena tiragem. Como nunca foi traduzido na íntegra ao português parece-nos uma justa homenagem, por ocasião do sesquicentenário de sua morte em $1^{\text {o. }}$ de fevereiro de 1855, apresentar esta publicação em língua portuguesa. A obra de Eschwege é conhecida e reconhecida no Brasil sobretudo por seu Pluto brasiliensis, uma sinopse da geologia econômica, tratando preferencialmente do ouro e dos diamantes, suas descobertas, mineração e legislação. Menos conhecida é sua obra puramente geológica, como observou Leonardos (1973:77): “Ainda não foi tentado um estudo em profundidade da obra geológica de Eschwege." Na última década foram traduzidos seus livros Jornal do Brasil (1818) e Brasil Novo Mundo (1824) que contêm algumas partes geológicas, diários de viagens, mas tratam também de assuntos etnográficos, botânicos e sócio-culturais, inclusive com participação de outros autores. Não cabe aqui apresentar mais uma biografia, as quais já existem várias (Choffat 1913; Sommer 1928, 1952; Leonardos 1973; Barbosa 1977; Renger 2002), nem tentar o estudo em profundidade clamado por Leonardos. Restringimo-nos a uma breve análise do citado Quadro Geológico, focando algumas facetas de sua obra geológica no contexto do estado de arte da geologia no início do século XIX.

Chegado a Alemanha em fins de 1821, depois de 18 anos longe da sua pátria, dos quais 11 no Brasil entre 1810 e 1821, Eschwege aproveitou uma licença para viajar através de alguns paises da Europa a fim de comparar a geologia do Brasil com a do Velho Mundo, finalizou o trabalho em abril de 1822 na cidade de Weimar. Lá reencontrou e noivou com seu amor de juventude, Sophie von Baumbach, agora uma dama da corte do Grão Duque Carl August de Sachsen-Weimar-Eisenach. Através destes laços familiares encontrou-se também com o famoso escritor e poeta Johann Wolfgang von Goethe (1749 - 1832), encarregado da supervisão das minas do principado e apaixonado geólogo amador, ao qual apresentou amostras de rochas do Brasil e por intermédio dele vendeu um número considerável de diamantes brutos que havia comprado com a permissão de D. Pedro I pela cotação oficial no Rio de Janeiro, bem como outras amostras mineralógicas para a coleção de pedras preciosas do Grão Duque. Nessa ocasião, a coleção foi entregue a Goethe como curador (Goethe 1949). Goethe anotou: "Uns cinqüenta cristais de diamantes brutos, peculiares isoladamente, e mais ainda quando observados em série, ora descritos e classificados conforme suas formas pelo Sr. Soret, me proporcionaram uma nova visão deste extraordinário e supremo fenômeno da natureza".

Ainda cheio de dúvidas se já chegara a hora de divulgar suas idéias sobre a geologia do Brasil, Eschwege publica seu Quadro geognóstico, uma brochura de 44 páginas. Em carta de $1^{\text {o. }}$ de junho de
1822, escreveu a José Bonifácio de Andrada e Silva, seu chefe em Portugal, entre 1803 e 1810: "Nada vi que se pudesse comparar com o Brasil e decidi-me a escrever um "Prospecto geognóstico do Brasil" que prometo remeter com o primeiro navio." (Barbosa 1977; original da carta no Arquivo Nacional, RJ). Eschwege se encontrou com Goethe diversas vezes o qual registrou a obra com elogios no seu diário, dando por resolvido a origem das rochas peculiares do Brasil. Parece que o trabalho foi originalmente escrito como uma espécie de nota explicativa para acompanhar as amostras incorporadas à coleção do Grão Duque, a quem também dedica o opúsculo.

A publicação de Eschwege chamou a atenção dos colegas europeus, como demonstra a tradução integral ao francês, publicada logo em 1823 nos Annales des Mines, sob o título "Esquisse géognostique du Brésil", bem como uma resenha "Note on geognosy of Brazil" no Philosophical Journal de Edinburgo. Somente em 1846 foi publicado um resumo de poucas páginas em português sob o título "Bosquejo geognóstico do Brasil" como aditamento da "Geologia elementar aplicada à agricultura", o primeiro livro didático de geologia impresso no Brasil, de Nérée Boubée, professor em Paris. Uma outra sinopse foi apresentada mais recentemente por Renger (1979).

O termo Geognosia foi criado em 1780 por Abraham Gottlieb Werner (1750 - 1817), o famoso professor da Academia de Minas de Freiberg na Saxônia, para designar uma ciência descritiva e de cunho aplicado ao contrário do termo Geologia proposto em 1778 pelo suíço Jean André de Luc (1727 - 1817), termo considerado por Werner mais teórico, filosófico, especulativo e, às vezes, fantasioso (Beringer 1954).

\section{GEOGRAFIAFÍSICA}

O Quadro geognóstico está dividido em duas partes. A primeira trata inicialmente da geografia física do Brasil, definindo a Serra das Vertentes e a Serra do Espinhaço como os grandes divisores de águas no Brasil, a primeira na direção leste - oeste entre as bacias do Rio Amazonas ao norte e o sistema Paraná/Paraguai ao sul e a segunda na direção meridiana. O naturalista José Vieira Couto (1752 - 1827) já havia reconhecido o importante papel desta serra que atravessa Minas Gerais do norte ao sul:

"Toda superfície da capitania de Minas Gerais é composta de continuados montes e serrotes, mas entre todos eles sobreleva-se muito esta dita serra, a qual eu lhe chamo a Grande Serra, como a mais principal, e que corta toda capitania do Sul ao Norte. Esta mesma serra pela sua cumeada, sempre encadeada, vai dividindo as águas da mesma Capitania em duas principais: em águas de Leste, que vão todas ao Rio Doce ... e em águas do Poente, que vão todas ao Rio de S. Francisco." (Couto 1801)

Eschwege, por sua vez, batizou esta cordilheira de Serra do Espinhaço e a define não somente como divisor de águas, mas identifica nela também um importante limite geológico e biogeográfico, incluindo também a 
Serra da Mantiqueira, estendendo-a assim até o Rio Grande do Sul. Posteriormente, Derby (1906) restringiu sua extensão ao segmento entre Ouro Preto e Juazeiro (Bahia), à margem do Rio São Francisco, passando por Minas Gerais, Bahia até o Sul de Pernambuco, praticamente coincidindo com a margem oriental da Bacia Sanfranciscana. Hoje considera-se a Serra das Cambotas, perto de Barão de Cocais, a terminação sul da Cordilheira do Espinhaço, concomitante à distribuição do Supergrupo Espinhaço. Sem nomear as serras, Eschwege já havia mencionado os grandes traços dos divisores das águas numa comunicação anterior (Eschwege 1817).

\section{ESTRATIGRAFIA}

A obra apresenta também uma primeira proposta de ordenação estratigráfica dos terrenos precambrianos no Brasil, notadamente de Minas Gerais, adotando a classificação proposta por Werner em 1787 e geralmente usada na Europa naquela época. Assim distingue quatro grandes divisões:

Primeira Formação Primitiva: granito, gnaisse e mica xisto; corresponde ao "embasamento cristalino";

Segunda Formação Primitiva: quartzo (lê-se quartzito), xisto argiloso, talco (xisto), formação ferrífera e pouco calcário; corresponde às seqüências supracrustais, notadamente os atuais supergrupos Rio das Velhas, Minas e Espinhaço;

Terceira Formação ou de Transição: xisto argiloso (ou ardósia), quartzo xisto, grauvaca e calcário maciço; é representada pelos grupos Macaúbas e Bambui;

Aluviões, cascalhos e a "tapanhoacanga" (isto é: canga).

É interessante registrar que, na mesma época, o naturalista José de Sá Bitencourt Câmara (1752 - 1828, natural de Caeté e irmão mais velho do Intendente Câmara), na sua "Memória mineralógica do terreno mineiro da Comarca de Sabará", escrita em 1822 (?) (Câmara 1897), propôs um esquema algo semelhante. Reconheceu o caráter estratificado da "Serra Principal", que "é o divisor geral das águas do Rio de São Francisco e Rio Doce", no centro de Minas Gerais. Identificou como principais camadas o xisto argiloso, a mina (isto é, o minério) de ferro especular micaceo, muitas vezes coberto até o cume por um conglomerado ferruginoso ("podingston que os naturais chamam Tapanhoacanga") e o "gré" (grès, isto é, arenito ou quartzito em francês). Na formação ferrífera chama atenção à jacutinga, um minério de ferro pulverulento, muitas vezes riquíssimo em ouro, que se estende desde de Morro do Pilar até os contrafortes da serra de Vila Rica e Mariana. Apesar de perceber uma seqüência cronológica das camadas: “ ... e apesar de serem todas quartzosas mostram ao Filósofo as diferentes épocas de sua criação", não preocupou de correlacionar-las com qualquer sistema europeu.

Mas não foi somente na nomenclatura estratigráfica que Eschwege seguiu os ensinamentos de Werner, pois nota-se no texto de Eschwege nitidamente uma interpretação netunista, apesar de que Eschwege nunca era aluno de Werner. As primeiras décadas do século XIX marcaram o auge da disputa entre o netunismo, do qual Werner foi o principal protagonista, e o plutonismo, defendido por James Hutton (1726 - 1796), naturalista e geólogo escocês. Os nomes derivam da mitologia: Netuno era o deus dos mares (ou das águas) dos romanos, Pluton o deus do inferno (ou do fogo) dos gregos. $\mathrm{O}$ netunismo explicava todas as rochas como precipitações a partir da água, baseado na história bíblica da criação da terra:

Aí Deus disse: Que a água que está debaixo do céu se ajunte num só lugar a fim de que apareça a terra seca! E assim aconteceu. Deus pôs na parte seca o nome de "terra" e nas águas que se haviam ajuntado ele pôs o nome de "mares". E Deus viu que o que havia feito era bom. (Gênesis I, 9-10).

Goethe era fervoroso adepto da teoria werneriana, que admitia a existência de vulcões, porém os considerava como um fenômeno subordinado, causado pela ignição de camadas carboníferas. Também o grande sábio Alexander von Humboldt $(1769$ - 1859) que estudou Geognosia com Werner em Freiberg, durante sua famosa viagem de pesquisas às Américas do Sul e Central (1799 - 1804), viu a geologia pelo ângulo do netunismo, mesmo escalando alguns vulcões como o Chimborazo no Equador e pesquisando no México, em 1803, o Popocatépetl, o Iztaccihuatl, o Jorullo, entre outros. Apenas em 1823, numa conferência perante a Academia de Ciências de Berlim, ele reconheceu o importante papel dos vulcões na formação da crosta terrestre, iniciando assim o fim da disputa (Engelhardt 2001). Trinta anos mais tarde, na releitura dos seus diários da viagem ao México anotou à margem "Anciennes folies neptuniennes!" [antigas loucuras netunísticas] (Leitner 2002). O próprio Eschwege parece ter se convertido também ao plutonismo, pois chamou sua obra principal sobre os recursos minerais e mineração no Brasil Pluto brasiliensis (1833). No próprio Quadro geognóstico faz referência à lava, quando compara as coberturas de canga, revestindo morros e encostas, com correntes de lava esfriada.

Eschwege e Humboldt encontraram-se em Paris no final de 1821, quando o último trabalhou no seu "Essai géognostique sur le gisement des roches dans les deux hémispheres" (Humboldt 1823), entre todas suas obras a mais geológica. Parece que houve uma inspiração mútua, e os dois tornaram-se amigos e, pelos menos a partir daquela época, correspondentes (Beck 1959). É interessante notar que Eschwege copiou à mão o esquema estratigráfico desse Essai, o "Tableau des Formations observées dans les deux Hémispheres" (por Alexandre de Humboldt 1822), que distingue cinco subdivisões estratigráficas, a saber: Terrenos Primitivos, de Transição, Secundários, Terciários e como quinta os Terrenos Vulcânicos (Acervo Eschwege do Arquivo Estadual de Marburg). 


\section{PETROGRAFIAE NOMENCLATURA}

Outra contribuição importante do "Quadro geognóstico do Brasil" é a definição de certos tipos de rochas desconhecidas na Europa, tais como itacolumito, especularita xisto (Eisenglimmerschiefer), itabirito (Eisenstein ou Eisenfels) e a tapanhoacanga, esta última hoje mais conhecida pelo nome de canga. Eschwege apresenta as rochas com seus principais componentes mineralógicos, ocorrência, distribuição, posicionamento geológico e indica a localidade-tipo. O nome Itabirito foi adotado pelo antigo arraial de Itabira do Campo, situado ao pé do "Morro de Itabira" ou Pico de Itabirito, em 1923, quando se emancipou de Ouro Preto.

Os principais litotipos do Brasil já foram apresentados pelo autor ao público alemão numa publicação anterior (Eschwege 1820), fazendo referência a um compêndio de geognosia que preparou para seus auxiliares e alunos no Brasil e deixou no Rio de Janeiro para ser impresso. Na realidade, nunca foi publicado, mas existe como manuscrito no acervo Eschwege do Arquivo Estadual de Marburg com o título Instrucções para os Mineiros e Officiaes Engenheiros que se houvessem de applicar à Administração das Minas; tomo $1^{\circ}$ Geognosia. Estranhamente, Eschwege não faz referência ao termo jacutinga, o itabirito pulverulento aurífero, na época assaz conhecido.

Os termos introduzidos por Eschwege se firmaram logo na bibliografia alemã (Cotta 1849) e foram usados por praticamente todos viajantes naturalistas que visitaram Brasil no século XIX. São válidos até hoje, não somente no vocabulário português, como são encontrados ainda na nomenclatura geológica internacional (Cagnacci-Schwicker 1968). O termo itacolumito criou alguma confusão na geologia do Brasil, sendo que Eschwege (1818) o definiu inicialmente como um "arenito de grão fino com uma matriz clorítica ..." que "em laminas finas é mais ou menos flexível ...", formando grandes serras em Minas e Goiás. No seu Quadro geognóstico usou o termo itacolumito também em sentido estratigráfico, juntando todos os quartzitos sob este nome, notadamente os do Quadrilátero Ferrífero e da Serra do Espinhaço. Mas Eschwege nem foi o primeiro a estudar o itacolumito; a primazia cabe a James Hutton que publicou em 1794 um pequeno tratado intitulado On the flexibility of the Brazilian stone pela Royal Society de Edinburgo. Parece que o fenômeno do quartzito flexível não é exclusivo no Brasil, pois Goethe lembra, no seu diário, da ocorrência de um "arenito articulado" na Silésia, no leste da Alemanha (Goethe 1949).

Derby (1906:374/5) já alertava sobre esta confusão: "Eschwege tinha uma teoria química-cristalina própria no que se refere ao modo de formação do que era considerado na sua época como rochas primitivas, entre as quais incluiu, como uma segunda divisão, as rochas arenosas e argilosas da Serra do Espinhaço. Não admitiu a sucessão de sedimentos entre elas, nem tentou subdividir-las em séries. O resultado mais infeliz deste ponto de vista, foi o de juntar todas as rochas quartzosas da região sob o nome geral de itacolumito, o que se tornou um pesadelo para os estudos da geologia do Brasil."

Nem por isso deixou Derby de outorgar a Eschwege o título "the founder of Brazilian geology". Atualmente entende-se como itacolomito (com 'o') o quartzito flexível (Guerra 1969; Leinz e Leonardos 1971), independente de sua posição estratigráfica ou local de ocorrência.

Com sua teoria sui generis das forças, que atuam a partir de certos centros de atração, Eschwege explica também a mudança de direção e mergulho das camadas, isto é, os fenômenos tectônicos. Em suas Contribuições à Geologia do Brasil (1832), ainda aguardando uma tradução completa ao português, o autor volta ao mesmo tema mais detalhadamente. A investigação das causas e regularidades de direção e mergulho das camadas dos sistemas orográficos dos dois hemisférios era um dos objetivos de Humboldt no seu Essai géognostique (1823), que podemos considerar como o início dos estudos tectônicos que levaram cerca de 150 anos até a consolidação da Tectônica das Placas.

\section{AROCHAMATRIZDOS DIAMANTES}

A segunda parte da obra trata da ocorrência dos diamantes no Brasil, especialmente em Minas Gerais, e de sua possível rocha matriz ou formadora. Baseado na ampla distribuição de rochas ferruginosas nas regiões diamantíferas e de minerais de ferro como satélites do diamante, e especialmente na existência de algumas amostras de canga limonítica com diamantes incrustadas, conclui que os diamantes se formaram na própria limonita. É interessante notar que naquela época amostras desse tipo eram extremamente raras, enquanto que hoje em dia são oferecidas, com certa freqüência, a colecionadores em Diamantina. Deve-se desconfiar que em algumas destas peças os diamantes tenham sido colados. A discussão sobre a verdadeira rocha matriz dos diamantes foi definitivamente solucionada somente com a descoberta de diamantes nas chaminés de kimberlito na África do Sul em 1872.

Apesar da - hoje evidente - inconsistência da argumentação de Eschwege, foram suas publicações e idéias geológicas sobre o Brasil que levaram ao primeiro achado de diamantes na Rússia. Alexander von Humboldt fez nos anos 1829/30 uma viagem científica à Rússia e à Sibéria, até a divisa chinesa. Antes de sua partida prognosticou, numa audiência com a czarina, a descoberta de diamantes, baseado nas analogias da geologia do Brasil e da Rússia. A equipe da expedição fez prospecções sistemáticas em todas as lavras aluvionares e, em julho de 1829, teve êxito na encosta ocidental (européia) das Montanhas Urais com a descoberta dos primeiros diamantes (Beck 1959).

Num estudo detalhado da ocorrência dos diamantes nas Montanhas Urais, Engelhardt (1830) compara a petrografia do itacolumito do Brasil, segundo Eschwege, com as rochas quartzíticas da Rússia, as quais chama também de itacolumito. Ao contrário de uma rocha matriz limonítica, como postula Eschwege no caso dos 
diamantes do Brasil, Engelhardt admite uma origem a partir do dolomito através de redução do carbonato sob altas temperaturas.

\section{CONCLUSÃO}

Apesar de decorridos mais de 180 anos desde a publicação do "Quadro geognóstico ..." do Barão de Eschwege, ainda falta um estudo da real importância desta obra, a qual apresenta assuntos ainda atuais da Geologia do Brasil. São ressaltados alguns aspectos tais como a estratigrafia precambriana e a geologia do diamante.

\section{AGRADECIMENTOS}

Manifesto meus agradecimentos aos colegas Carlos Alberto Rosière pela criteriosa revisão, Maria Márcia Magela Machado pelo tratamento das imagens e Antônio Carlos Pedrosa Soares pelo incentivo à publicação deste trabalho e da tradução.

\section{REFERENNCIAS}

Barbosa, W. de A. 1977 Barão de Eschwege. Casa de Eschwege, Belo Horizonte, 74 pp.

Beck, H. 1959 Wilhem Ludwig von Eschwege und Alexander von Humboldt. in: Alexander von Humboldt 1769 - 1859. Akademie-Verlag, Berlin, pp. 37-68

Beringer, C. 1954 Geschichte der Geologie und des geologischen Weltbildes. Enke, Stuttgart, 158 pp.

Boubée, N. 1846 Geologia elementar applicada à Agricultura com hum diccionario dos termos geologicos.: Typographia Nacional, Rio de Janeiro, 134+55+127 pp.

Cagnacci-Schwicker, A. 1968 International Dictionary of Metallurgy, Mineralogy, Geology. McGraw-Hill, New York, $1530 \mathrm{pp}$.

Câmara, J. de S. B. 1897. Memória mineralógica do terreno mineiro da Comarca de Sabará. Rev. Arqu. Públ. Mineiro, 2:599-609

Choffat, P. 1913 Le baron d'Eschwege (1777-1855). - Comm. Serv. Geol. Portugal, t. 11:181-214

Cotta, B. 1849 Leitfaden und Vademecum der Geognosie. Arnold, Dresden, 291 pp.

Couto, J. V. 1801 Memória sobre as minas da capitania de Minas Gerais - Manuscrito do Instituto Histórico-Geográfico Brasileiro [publicado Rio de Janeiro: Laemmert, 1842, reimpresso em: Rev. Arqu. Públ. Mineiro, 6:55-106; 1900)]

Derby, A. O. 1906. The Serra do Espinhaço. Journal of Geology, 14: $374-401$

Engelhardt, M. von. 1830. Die Lagerstätte der Diamanten. Annalen der Physik und Chemie, 20:524-539

Engelhardt, W. von. 2001. Goethe und Alexander von Humboldt: Bau und Geschichte der Erde. HiN, Humboldt im Netz, v. II/ 3, 9 pp. (www.uni-potsdam.de/u/romanistik/ humboldt/hin)

Eschwege, W.L. von. 1817. Idées génerales sur la constituition géologique du Brésil. Annales des Mines (Paris), 2. série, t. 2:238-240

Eschwege, W.L. von. 1818 Vorkommen des elastischen Sandsteins. Annalen der Physik, 58:99-101
Eschwege, W.L. von. 1820. Über einige merkwürdige brasilianische Gebirgsformationen Annalen der Physik, 65:414-420

Eschwege, W.L. von. 1818 Journal von Brasilien, oder vermischte Nachrichten aus Brasilien, auf wissenschaftlichen Reisen gesammelt. - Weimar (Landes-Industrie-Comptoir) 1818, 2 vol. (Bertuchs Neue Bilbliothek der wichtigsten Reisebeschreibungen ...etc., v. 14 e 15) em portugues: Jornal do Brasil, 1811 - 1817 ou informes .... Belo Horizonte (Fundação João Pinheiro, Centro de Estudos Históricos Culturais) 2002, 408 pp.

Eschwege, W.L. von. 1820 Nachrichten aus Portugal und dessen Colonien, mineralogischen und bergmännischen Inhaltes. Ein Seitenstück zum Journal von Brasilien. G.C.E. Meyer, Braunschweig, 274 pp.

Eschwege, W.L. von. 1822 Geognostisches Gemälde von Brasilien und wahrscheinliches Muttergestein der Diamanten. Landes-Industrie-Comptoir, Weimar, 44 pp.

Eschwege, W.L. von. 1823. Esquisse géognostique du Brésil, suivie d'une dissertation sur la gangue originaire du diamant; extrait traduit de 1'allemand par M. Combes. Annales des Mines (Paris), 3e. série, t. 8:401-430

Eschwege, W.L. von. 1823. Note on geognosy of Brazil. Philosophical Journal (Edinburgh), 9:200-202

Eschwege, W.L. von. 1824. Brasilien, die Neue Welt ... während eines elfjährigen Aufenthaltes von 1810 - 1821 beobachtet ... Friedrich Vieweg, Braunschweig 2 vol.; em português: Brasil, novo mundo. Belo Horizonte (Fundação João Pinheiro, Centro de Estudos Históricos Culturais) v. 1, 1996; v. 2, 2001

Eschwege, W.L. von. 1832. Beiträge zur Gebirgskunde Brasiliens. Reimer, Berlim, 488 pp.

Eschwege, W.L. von. 1833. Pluto brasiliensis. Berlim: Reimer, 622 p.; tradução em português de D. de F. Murta: Pluto brasiliensis. Itatiaia/Edusp, Belo Horizonte/São Paulo, 2 vol.

Eschwege, W.L. von. 1846. Bosquejo geognostico do Brasil, com uma dissertação sobre a matriz dos diamantes. - in: Boubée, N. Geologia elementar. Typographia Nacional, Rio de Janeiro, pp. 35-40

Goethe, J. W. von. 1949. Schriften zur Geologie und Mineralogie 1812 - 1832. (ed. G. Schmid) Böhlau, Weimar, 438 pp.

Guerra, A. T. 1969. Dicionário geológico-geomorfológico. IBGE, Rio de Janeiro, 439 pp.

Humboldt, A. von. 1823. Essai géognostique sur le gisement des roches dans les deux hémispheres. Levrault, Paris, 379 pp. [não consultado]

Hutton, J. 1794. On the flexibility of the Brazilian stone. Trans. R. Soc. Edinburgh, 3:86-94 [não consultado]

Leinz, V. \& Leonardos, O.H. 1971. Glossário geológico. EdUSP, São Paulo, 236 pp.

Leitner, U. 2002 “Anciennes folies neptuniennes!” Über das wiedergefundene "Journal de Mexique à Veracruz" ... de A.von Humboldt. HiN, Humboldt im Netz, v. III/5; 13 pp. (www.unipotsdam.de/u/romanistik/ humboldt/hin)

Leonardos, O.H. 1973. Geociências no Brasil: a contribuição germânica. Fórum-Sulina, Rio de Janeiro, 345 pp.

Renger, F. 1979. Evolução dos conceitos geológicos da Serra do Espinhaço. In: SBG/Núcleo MG, Bol. 1:9-27

Renger, F.E. 2002. Eschwege, o brasilianista. in: Eschwege, W.L. von: Jornal do Brasil, 1811 - 1817; tradução de F.E. Renger et al.; Fundação João Pinheiro, Belo Horizonte, 2002, 408 pp.

Sommer, F. 1928. Wilhelm Ludwig von Eschwege (1777 - 1855). Ausland und Heimat, Stuttgart, 190 pp.

Sommer, F.1952. Guilherme Luis, Barão de Eschwege - Patriarca da Geologia Brasileira. Melhoramentos, São Paulo, 120 pp. 